If the effect is definitely established, then the partnership of space and time cannot be upheld in the general relativity theory. If the effect is shown not to exist, it will provide independent evidence of the validity of the general relativity principle.

W. R. Mason.

180 Varick Street, New York City.

${ }^{1}$ Kennedy, "The Velocity of Light", NATURE, 130, 277, Aug. 20, 1932.

'McVittie, "Dirac's Equation in General Relativity", Mon. Not. Roy. Astro. Soc., 82, 868-877, Oct. 1932.

Mason. A Newtonian Gravitational System and the Expanding Universe", Phil. Mag., Ser. 7, 14, 386, Sept., 1932.

\section{Ionisation Density and Critical Frequency}

IN some recent papers ${ }^{1}$ on ionisation in the upper atmosphere, the theoretical relation between ionisa. tion density $N$ and eritical frequency $f$ has been taken to be $N=(3 / 2)\left(\pi m / e^{2}\right) f^{2}$. On the other hand, J. J. Thomson has, on various occasions, as have $\mathrm{L}$. Tonks and I. Langmuir ${ }^{2}$ published formulæ identical with this except that the coefficient $3 / 2$ is replaced by unity.

An examination of the origin of the difference reveals that the numerical factor is different from unity when the displacement of negative relative to positive charges in the medium gives rise to a doublet field in addition to the impressed field. This is the force $a P$ in Lorentz's expression $E+a P$ for the force acting on an electron ${ }^{3}$. Such a 'polarisation' force can only exist, however, when there is some detailed arrangement of the negative with respect to the positive charges, so that a volume element of the polarised medium is distinguishable from a volume element of the unpolarised medium.

In an ionised gas, the only possible arrangement of this type is that associated with the Debye-Hückel ion cloud, but its effect is completely negligible 4 . The 'polarised' gas contains no electric doublets and in an infinite gas only the space charge developed in the volume will contribute to the force acting on any ion. Consequently, the relation of electron concentration to critical frequency is $N=\left(\pi m / e^{2}\right) f^{2}$ in this case.

General Electric Company, Schenectady. May 24.

1 Among others, Proc. Phys. Soc., 45, 389; 1933.

"Phys. Rev., 33, 195; 1929.

"Theory of Electrons", p. 139

- Phys. Rev., 38, 1219; 1931.

\section{Catalysis of the Hydrogen-Sulphur Reaction by Minute Traces of Oxygen}

Is has been pointed out ${ }^{1}$ that the curvature found by Norrish and Rideal ${ }^{2}$ in the $\left.\log \frac{d\left[\mathrm{H}_{2} \mathrm{~S}\right]}{d t}\right|_{T} ^{1}$ curve representing the hydrogen-sulphur reaction was only to be observed in the presence of nitrogen. Pending further inquiry, we tentatively ascribed this to a facilitation by the nitrogen molecules of some chain mechanism. The investigation of this point, now complete, indicates that the effect is foreign to the true reaction, and arises from minute traces of oxygen present in the hydrogen-nitrogen mixtures.

Originally these mixtures had been prepared from pure hydrogen ard cylinder nitrogen washed with alkaline pyrogallol, and, as a further precaution, treated after mixing with the same reagent. Since certain tests had indicated that minute traces of oxygen in otherwise pure hydrogen considerably accelerated the velocity of hydrogen sulphide forma. tion at the lower temperatures, it seemed worth while to examine the effect of an even more rigorous exclusion of oxygen from the mixed gases. Extensions of the pyrogallol treatment diminished the velocity until it eventually became practically identical with that resulting from the use of pure hydrogen. It was, however, felt that even freshly prepared pyrogallol might possibly lead to a contamination of the gases with traces of carbon compounds, and, therefore, in a parallel series of experiments this reagent was replaced by chromous chloride together with amalgamated zinc. Again, values approximating to those for pure hydrogen were obtained. Specimen results from the homogeneous reaction in bulbs are given below :-

\begin{tabular}{|c|c|c|c|c|}
\hline & \multicolumn{3}{|c|}{ Rate of formation of $\mathrm{H}_{2} \mathrm{~S}$ (gm. per sec. $\left.\times 10^{-8}\right)$} \\
\hline Temp. & $\begin{array}{c}\mathrm{H}_{2}+\mathrm{N}_{3} \\
\text { normal }\end{array}$ & $\begin{array}{c}\mathrm{H}_{\mathbf{2}}+\mathrm{N}_{2} \text { over } \\
\text { pyrogallol }\end{array}$ & $\begin{array}{c}\mathrm{H}_{2}+\mathrm{N}_{2} \text { over } \\
\text { CrCH }_{2}+\mathrm{Zn}\end{array}$ & Pure $\mathrm{H}_{2}$ \\
\hline $291^{\circ}$ & $2 \cdot 51$ & $1 \cdot 28$ & 1.17 & 1.13 \\
\hline
\end{tabular}

The effect of so minute a quantity of oxygen is remarkable and unexpected, but there seems little doubt that it accounts for the form assumed by the $\log$ velocity $1 / T$ plots of Norrish and Rideal and thus invalidates deductions made therefrom as to the nature, temperature coefficient or heat of activation of the reactions.

The first stage of the investigation has now been completed and will be published in full shortly. E. E. Aynsley. T. G. Pearson.

University of Durham, Armstrong College,

Newcastle-upon-Tyne. June 2.

1. Aynsley, Pearson and Robinson, Nature, 131, 471, April 1, 1933. ${ }^{2} J$. Chem. Soc., 123, 696; 1923.

Reducing Bodies, and Fumarase, in Tumours

Wrixh reference to Dr. L. J. Harris's comments in NATURE of July 1 (p. 27) on the presence of reducing bodies in tumours, perhaps the following observations may be of some interest. In making a systematic investigation of the distribution of fumarase in animal and plant tissues, I have studied the activity of several human tumours. They have all shown very powerful fumarase activity and usually more so than the surrounding host tissue. In performing the test for fumarase activity, I have estimated the $l$-malic acid formed by adding acetic acid and concentrated ammonium molybdate solution, filtering off the proteins, etc., and examining the resulting clear solution by means of the polarimeter. With all tumours so far examined, the solution after adding acetic acid and molybdate became deeply coloured, probably due to the formation of the lower oxides of molybdenum and indicating the presence of reducing bodies in the tumours. The host tissues failed to give such extensive reduction of the molybdic acid reagent.

Biochemical Laboratory, J. H. QUASTEL.

Cardiff City Mental Hospital. July 3 . 